\title{
An electronic trigger tool to optimise intravenous to oral antibiotic switch: a controlled, interrupted time series study
}

\author{
Marvin A. H. Berrevoets ${ }^{1 *}$, Johannes (Hans) L. W. Pot ${ }^{2}$, Anne E. Houterman², Anton (Ton) S. M. Dofferhoff ${ }^{3}$, \\ Marrigje H. Nabuurs-Franssen ${ }^{4}$, Hanneke W. H. A. Fleuren², Bart-Jan Kullberg ${ }^{1}$, Jeroen A. Schouten ${ }^{5}$ \\ and Tom Sprong ${ }^{3}$
}

\begin{abstract}
Background: Timely switch from intravenous (iv) antibiotics to oral therapy is a key component of antimicrobial stewardship programs in order to improve patient safety, promote early discharge and reduce costs. We have introduced a time-efficient and easily implementable intervention that relies on a computerized trigger tool, which identifies patients who are candidates for an iv to oral antibiotic switch.

Methods: The intervention was introduced on all internal medicine wards in a teaching hospital. Patients were automatically identified by an electronic trigger tool when parenteral antibiotics were used for $>48 \mathrm{~h}$ and clinical or pharmacological data did not preclude switch therapy. A weekly educational session was introduced to alert the physicians on the intervention wards. The intervention wards were compared with control wards, which included all other hospital wards. An interrupted time-series analysis was performed to compare the pre-intervention period with the post-intervention period using '\% of i.v. prescriptions $>72 \mathrm{~h}$ ' and 'median duration of iv therapy per prescription' as outcomes. We performed a detailed prospective evaluation on a subset of 244 prescriptions to evaluate the efficacy and appropriateness of the intervention.
\end{abstract}

Results: The number of intravenous prescriptions longer than $72 \mathrm{~h}$ was reduced by $19 \%$ in the intervention group $(n=1519)(p<0.01)$ and the median duration of iv antibiotics was reduced with 0.8 days $(p=<0.05)$. Compared to the control group $(n=4366)$ the intervention was responsible for an additional decrease of $13 \%$ $(p<0.05)$ in prolonged prescriptions.

The detailed prospective evaluation of a subgroup of patients showed that adherence to the electronic reminder was $72 \%$.

Conclusions: An electronic trigger tool combined with a weekly educational session was effective in reducing the duration of intravenous antimicrobial therapy.

Keywords: Decision support system, Antimicrobial stewardship, Iv-oral switch, Quality of care

\footnotetext{
* Correspondence: Marvin.Berrevoets@radboudumc.nl

${ }^{1}$ Department of Internal Medicine and Infectious Diseases, Radboudumc, Nijmegen, the Netherlands

Full list of author information is available at the end of the article
} 


\section{Background}

Most patients with an infection that requires inpatient treatment initially receive empirical intravenous (iv) antimicrobial therapy. When the patient clinically improves within $48 \mathrm{~h}$ and results from microbiology cultures and other tests become available these iv antibiotics may be switched to oral therapy, with the exception of certain clinical conditions that necessitate prolonged iv treatment (e.g. Staphylococcus aureus bacteremia, endocarditis, meningitis). Previous studies have shown that a timely switch from intravenous to oral therapy is safe and reduces risk of complications related to intravenous treatment, healthcare costs and duration of hospitalization [1-6].

A Dutch study found that for patients with communityacquired pneumonia, a switch to oral antibiotics was possible in $46 \%$ of the patients on day 3 of treatment, but was not performed in $40 \%$ of eligible switch opportunities [7]. Barriers that preclude switching include misconceptions, practical considerations and organizational factors $[7,8]$. Different approaches have been deployed to incorporate switch therapy into daily practice. However, the major conversion programmes evaluated in the literature were labour intensive interventions [3, 4] and not aimed at solving the barriers to timely switching therapy from iv to oral [5]. Previous research showed that introducing switch therapy into daily practice by a computerized trigger tool was effective in promoting iv to oral switch therapy $[9,10]$. However, these reminders were produced with low specificity, were only performed for a limited number of antibiotics or had a low adherence rate.

The goal of this study was to evaluate the effect of a combined intervention targeting different barriers that preclude switching. The first intervention, a computerized reminder, was chosen to target both practical and organisational factors, while continuous education and feedback on iv-oral switch practice and promoting the use of a so called 'switch card' aimed to improve misconceptions about switching. The computerized reminder relies on an electronic trigger tool, which identifies patients who are candidates for antibiotic switch therapy.

With this combined intervention we aimed to improve the rate of safe iv to oral antibiotic switch on internal medicine wards.

\section{Methods}

This controlled intervention study was performed at the Canisius-Wilhemina Hospital, Nijmegen, the Netherlands, a 455-bed non-academic teaching hospital. The study was performed during a 26-month period. The pre-intervention period was defined as the first 13 months of the study period and the post-intervention period was defined as the last 13 months of the study period. The intervention was carried out at the internal medicine wards. The control wards included all other hospital wards.

\section{Intervention}

\section{Computerised reminders}

The multidisciplinary Antimicrobial Stewardship team (AST) (comprised of an infectious disease specialist, microbiologist and clinical pharmacist) created an automatic warning system that identifies candidates who are eligible for iv-oral switch therapy. The tool was based on the Dutch National Antibiotic Switch guidelines [3] and was developed in Crystal Reports ${ }^{\bullet}$ using data from the hospital pharmacy database and the clinical chemistry department.

Patients were identified as eligible for iv-oral switch therapy when antibiotic treatment had been prescribed 48 to $72 \mathrm{~h}$ previously. To alert the physician of the possibility of iv-oral switch an automated reminder was sent on day 3 of the treatment. This trigger tool automatically checked whether the following clinical or pharmacological conditions precluded switch therapy: (i) increase in CRP during iv treatment; (ii) neutrophils $<0,5 * 10^{9} / \mathrm{ml}$; (iii) leukocytes $<1^{*} 10^{9} / \mathrm{ml}$; (iv) usage of parenteral medication only or total parenteral nutrition, as an inability for oral intake predictor; (v) specific or high dose antibiotic suggesting severe infection (e.g. endocarditis, meningitis) (Additional file 1: Appendix 1). Patients meeting one or more of these criteria were not selected for switch therapy. If a single patient received more than one antibiotic, each single prescription (given for more than $48 \mathrm{~h}$ ) was checked by the trigger tool to determine eligibility for iv-oral switch. Every morning, for each eligible patient, a written notice was automatically generated and sent out to the department secretary. This form contained identifying data of the patient, the antibiotic(s) that were eligible to be switched, and possible oral options or alternatives for these antibiotics. The form was presented to the attending physician during ward rounds by the department secretary. The decision whether to switch or not was made by the physician, based on the clinical situation of the patient. For each patient the physician was asked to fill in a form on which considerations concerning the switch were listed (ability to switch, impeding reasons). Reasons precluding iv-oral switch were categorized as 'clinical instability', no oral intake possible, severe infection (e.g. Staphylococcus aureus bacteremia, meningitis, endocarditis) or resistant micro-organisms for which no oral therapy is available.

A second reminder was sent when the iv medication order was started 96 to $120 \mathrm{~h}$ before and no oral switch had yet been performed, based on the same algorithm as mentioned earlier. To prevent alert fatigue a maximum of two reminders per medication order per patient was chosen.

Due to organizational factors no switch forms were distributed during the weekend.

\section{Educational program}

The start of the intervention was preceded by an educational program. Pocket cards with the switch protocol 
were presented to each physician. The hospital protocol (Additional file 2: Appendix 2) recommends to choose an oral antibiotic based on culture results. When no oral formulation of the iv antibiotic eligible for switch is available and culture results are negative, an oral antibiotic covering a similar spectrum as the empirically started iv antibiotic is recommended (e.g. ceftriaxon should be switched to oral amoxicillin/clavulanic acid).

To optimize adherence, direct feedback and education was given to the physicians working on the intervention wards regarding the returned switch forms in a weekly short meeting.

During this 15-min meeting (called "Switch of the week") one or more of the completed switch forms were selected by an infectious disease specialist (T.S.) and the content was presented to the physicians. Both appropriate and inappropriate decisions of the physicians were discussed and feedback was given on the decisions made.

\section{Prospective observational study to assess the effectiveness of the intervention}

To determine the effectiveness of the intervention in promoting iv-oral switch therapy, data of all iv antibiotic prescriptions was collected from the hospital pharmacy database for the whole study period. The intervention group was represented by patients from the internal medicine wards, while the control group was represented by patients in the same hospital during the same period from all other wards. The intervention was carried out at the internal medicine wards during the last 13 months of the study period. The percentage of intravenous prescriptions that exceed $72 \mathrm{~h}(\%>72 \mathrm{~h})$ and median treatment duration in days of intravenous therapy on the intervention wards were calculated and compared to the control period and the control wards.

\section{Detailed prospective evaluation of a subset of prescriptions to assess appropriateness}

During the first 4 months of the intervention period, antibiotic prescriptions selected by the trigger tool were included in a detailed analysis to evaluate the efficacy and appropriateness of the intervention. The following patient data were collected to categorize the switch: patient's age and gender, source of infection, time of hospitalization, antimicrobial treatment, starting date, dose, body temperature, blood leukocyte count, blood neutrophil count, culture results and reasons which impeded the switch, based on the returned switch-forms. The appropriateness of the iv to oral switch was evaluated by an infectious disease specialist (TS). Options to categorize the conversion were: 'appropriate switch', 'inappropriate switch', 'appropriate continuation of iv therapy' and 'inappropriate continuation of iv therapy.
Categorization was based on the following switch criteria: no indication for prolonged iv antibiotic treatment (e.g. Staphylococcus aureus bacteremia, endocarditis, meningitis), improving vital signs, susceptible micro-organism (if cultured) for oral antibiotics, and the presence of a functional tractus digestivus. A patient who fulfilled these 4 switch criteria and who was switched to oral antibiotic therapy was categorized as an appropriate switch (Additional file 2: Appendix 2 - Table S3).

\section{Endpoints}

The primary endpoints were the change in percentage of iv treatment exceeding $>72 \mathrm{~h}$ for all patients in both the intervention group and control group between the pre- and post-intervention period and the percentage of patients appropriately switched to oral therapy on day 3 of treatment. Secondary outcome was the change in median treatment duration.

\section{Statistics}

All data was analyzed using the IBM SPSS20 software package. We performed an interrupted time series analysis (ITSA), for which we used segmented linear regression to assess the significance of changes in level and slope of the regression lines before and after the introduction of the intervention for both the intervention and control groups $[11,12]$. This methodology evaluates data collected at multiple time points before and after an intervention to detect whether the intervention had a greater effect than the expected secular trend. An abrupt intervention effect constitutes a change in the level of the outcome directly after the intervention is implemented. The slope represents a gradual change in the outcome parameter during the segment [12]. We divided the dataset into monthly periods (of which there were 26; 13 periods before the intervention started and 13 periods after the intervention started). The analysis was performed for the outcomes percentage of iv treatment $>72 \mathrm{~h}$, and median treatment duration in both the intervention and control group. We used a $p$-value of $<0.05$ as a threshold for all statistical tests.

Adherence to the intervention was determined by dividing the prescriptions that were appropriately switched (numerator) by the total amount of prescriptions eligible for switch (denominator).

\section{Ethics statement}

As iv to oral antibiotic switch therapy is proven to be safe and effective and is an essential element of many antimicrobial stewardship programs we considered timely switch as a standard of care, and our study is an evaluation of a new method to promote this standard of care. Patient data were collected anonymously. This study does not fall within the remit of the Medical Research involving 
Human Subjects Act (WMO). Therefore the study can be carried out in the Netherlands without an approval by an accredited research ethics committee and without explicit informed consent of the participants.

\section{Results}

\section{Baseline characteristics}

During the whole study period, 1519 patients on the intervention wards and 4366 patients on the control wards received intravenous antibiotics (Table 1). At baseline, on the intervention wards $50.5 \%$ of prescriptions were given for $>72 \mathrm{~h}$ versus $49.8 \%$ on the control wards.

\section{Prospective observational study to assess the effectiveness of the intervention}

Using interrupted time-series analysis, we compared the percentage of iv antibiotic prescriptions $>72 \mathrm{~h}$ in the pre-intervention and post-intervention periods. There was a significant decrease (reduction $19.3 \%, p<0.001$; Table 2 , Fig. 1) in the intervention group. We observed a significant but smaller decrease in the control group as well (reduction 6.1\%, $p<0.05$; Table 2, Fig. 1). The difference between the intervention and control group showed a significant additional reduction of intravenous antibiotic usage of $13.2 \%$ $(p=0.014)$ in favour of the intervention group.

During the post-intervention period, a non-significant increase in \% of prescriptions $>72 \mathrm{~h}$ was observed in the intervention-group (solid line in the white area, slope of $+0.48 \%$ / month, $p=0.43$; Fig. 1) and a non-significant decrease in the control group (dashed line in the white area, slope of $-0.26 \% /$ month, $p=0.46$; Fig. 1 ), with no significant difference between these groups.

Median duration of antibiotic usage on the intervention wards was decreased by 0.8 day ( 4.0 to $3.2, p=0.015$ ) (Table 2) after the intervention was introduced. There was no significant decrease observed on the control wards or between the intervention and control wards.

\section{Detailed prospective evaluation of a subset of prescriptions to assess appropriateness}

During a 4-month period there were 244 unique antibiotic medication orders for which the intervention was activated, 21 prescriptions were excluded, since these patients left the hospital on the day of the possible ivoral switch. This resulted in 223 medication orders for a detailed analysis (Fig. 2).

Of all medication orders, 116 were eligible for switch. Of these, 84 were switched correctly (72\%) and $32(28 \%)$ were incorrectly not switched. A total of 78 orders were not eligible for switch. For 29 orders there was no evaluation possible, either because the switch order was generated in the weekend or there was no form filled in. Most of the patients with medication orders that were candidates for conversion had pneumonia (24.7\%). The mean age was 71 years and most patients were female (53\%). Table 3 shows data about the infections treated, and antibiotics prescribed during this phase of the study. The antibiotic agent that was most often eligible for switch was cefotaxime (27.5\%).

During the detailed prospective evaluation, the digital switch forms were generated at two points in time. In case the first form did not result in a switch, a second form was generated from 96 to $120 \mathrm{~h}$ after therapy initiation. This resulted in 56 switch forms generated of which 20 lead to a correct switch and no incorrect switches, 31 were not eligible for iv-oral switch and 5 were indicated as incorrectly not switched (Fig. 2).

This detailed analysis showed that no patients were inappropriately switched.

\section{Discussion}

A computerized intervention in combination with an educational and feedback program, safely reduces the percentage of prolonged intravenous antibiotic use in hospital wards with $19 \%$.

Many studies have demonstrated that iv-oral switch therapy is associated with a reduced length of iv therapy and low clinical failure rate [6]. In most studies an investigator identified cases eligible for switch by manual data extraction. This is time consuming and has limited feasibility in daily practice.

In this study, a simple automated trigger tool in our clinical pharmacy database, selected patients eligible for switch. An automated reminder was printed and sent to the ward prompting the physician to perform an iv-oral switch.

Table 1 Baseline characteristics of patients on the intervention wards and controls wards during the pre-intervention and post-intervention periods

\begin{tabular}{|c|c|c|c|c|}
\hline \multirow[t]{2}{*}{ Characteristic } & \multicolumn{2}{|l|}{ Intervention wards } & \multicolumn{2}{|l|}{ Control wards } \\
\hline & Pre-intervention period & Post-intervention period & Pre-intervention period & Post-intervention period \\
\hline Patients & 771 & 748 & 2154 & 2212 \\
\hline Hospital admissions & 880 & 835 & 2458 & 2478 \\
\hline Medication orders (iv antibiotics) & 1781 & 1534 & 4769 & 4294 \\
\hline Mean age [range] & $68.3[16-101]$ & $68.4[17-101]$ & $54.8[1-100]$ & $54.1[0-98]$ \\
\hline Female (\%) & 405 (53\%) & $371(50 \%)$ & $1029(48 \%)$ & $1073(49 \%)$ \\
\hline
\end{tabular}


Table 2 Change in percentage of iv antibiotic prescriptions $>72 \mathrm{~h}$ and median antibiotic duration (days) during the pre-intervention and post-intervention period and comparison between the intervention and control group using interrupted time series analysis

\begin{tabular}{|c|c|c|c|c|c|c|}
\hline & $\begin{array}{l}\text { Slope during the pre- } \\
\text { intervention period (SE) }\end{array}$ & $P$ & $\begin{array}{l}\text { Change in level after } \\
\text { intervention }^{a} \text { (SE) }\end{array}$ & $P$ & $\begin{array}{l}\text { Slope during the post- } \\
\text { intervention period }{ }^{\mathrm{b}} \text { (SE) }\end{array}$ & $P$ \\
\hline \multicolumn{7}{|c|}{ Percentage of iv antibiotic prescriptions $>72 \mathrm{~h}(\%)$} \\
\hline Intervention group & $-0.03(0.42)$ & 0.94 & $-19.30(4.41)$ & $<0.01$ & $0.48(0.59)$ & 0.43 \\
\hline Control group & $-0.36(0.25)$ & 0.16 & $-6.12(2.61)$ & $<0.05$ & $-0.26(0.35)$ & 0.46 \\
\hline $\begin{array}{l}\text { Difference between intervention } \\
\text { and control group }\end{array}$ & $0.33(0.48)$ & 0.50 & $-13.17(5.13)$ & $<0.05$ & $0.74(0.68)$ & 0.29 \\
\hline \multicolumn{7}{|l|}{ Median duration of iv antibiotics (days) } \\
\hline Intervention group & $-0.02(0.03)$ & 0.49 & $-0.77(0.29)$ & 0.015 & $0.02(0.04)$ & 0.63 \\
\hline Control group & $-0.38(0.03)$ & 0.16 & $-0.15(0.28)$ & 0.59 & $0.04(0.04)$ & 0.32 \\
\hline $\begin{array}{l}\text { Difference between intervention } \\
\text { and control group }\end{array}$ & $0.02(0.04)$ & 0.62 & $0.62(0.41)$ & 0.14 & $-0.02(0.05)$ & 0.72 \\
\hline
\end{tabular}

${ }^{a}$ Measures the immediate impact of the intervention

${ }^{\mathrm{b}}$ Measures the long-term impact over time of the intervention

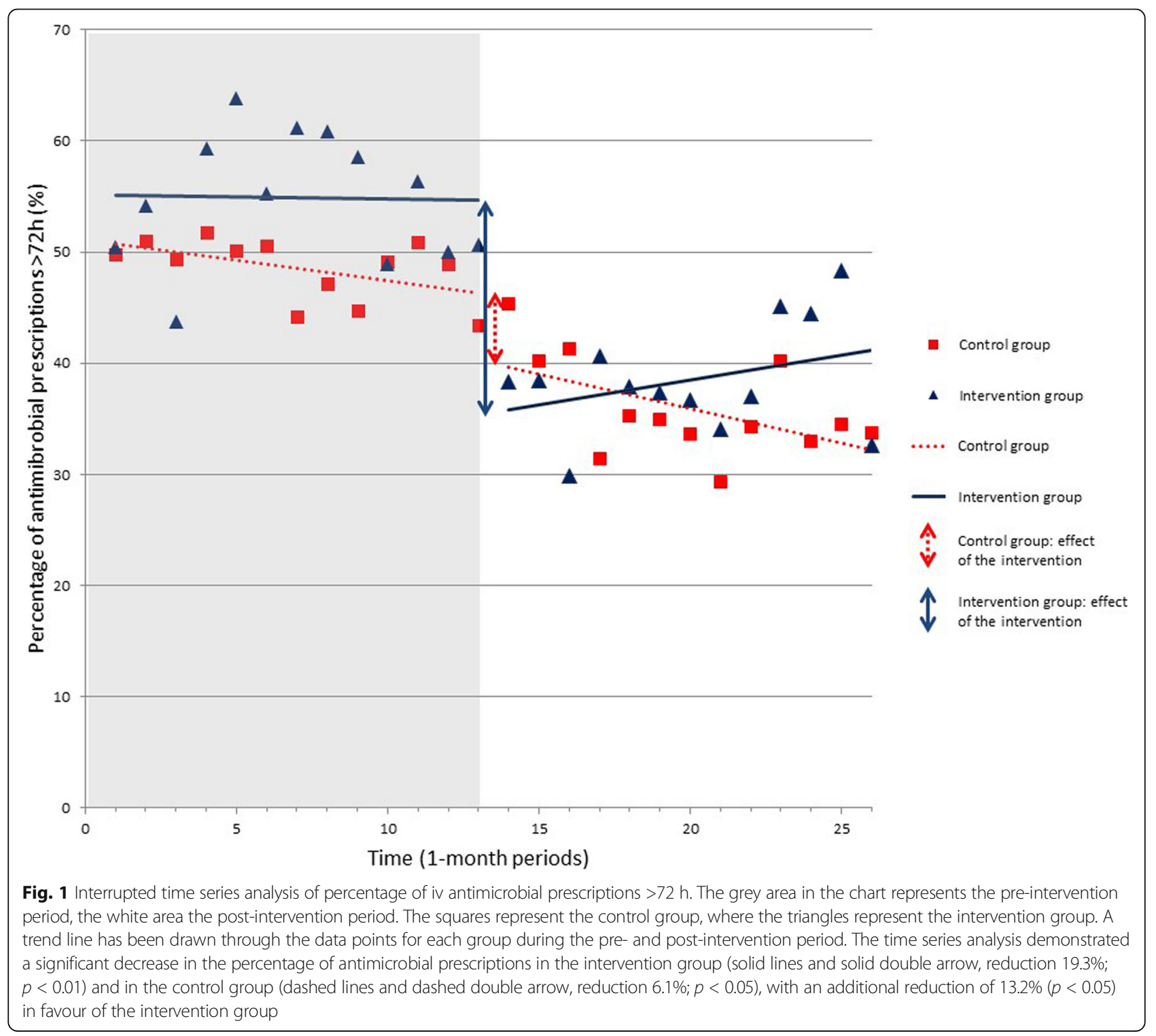




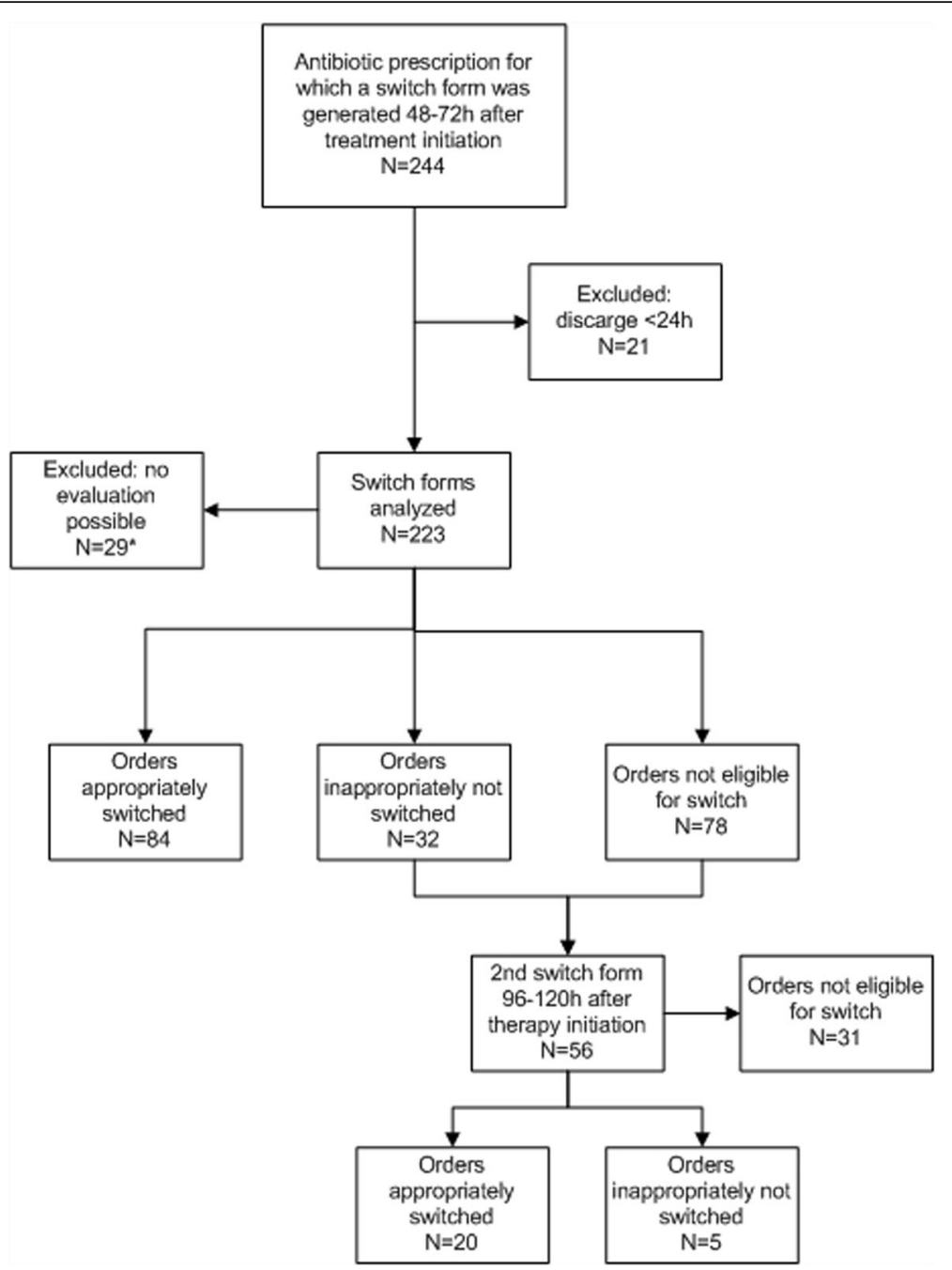

Fig. 2 Prospective evaluation of a subset of prescriptions. * no switch form returned or switch form generated during weekend days

Table 3 Detailed prospective analysis; indications and antibiotics prescribed for 244 analysed switch forms

\begin{tabular}{llll}
\hline Indication & \multicolumn{3}{c}{ Antibiotic } \\
\hline Pneumonia & $24.7 \%$ & cefotaxim & $27.5 \%$ \\
Sepsis & $20.4 \%$ & ciprofloxacin & $19.0 \%$ \\
Pyelonephritis/urosepsis & $18.6 \%$ & amoxicillin & $16.2 \%$ \\
Abdominal infection & $17.6 \%$ & metronidazole & $15.0 \%$ \\
Skin and soft tissue infection & $11.5 \%$ & cefazolin & $12.1 \%$ \\
Unknown indication & $2.5 \%$ & penicillin & $4.5 \%$ \\
Gastro-enteritis & $2.5 \%$ & clindamycin & $4.0 \%$ \\
Bone and joint infection & $1.1 \%$ & fluconazole & $0.8 \%$ \\
Other & $1.1 \%$ & ceftriaxone & $0.4 \%$ \\
& & flucloxacillin & $0.4 \%$ \\
\hline
\end{tabular}

By automating the intervention, we were able to systematically reduce workload, which was a problem in many other studies [13]. In addition, we gave direct feedback to the participating physicians improving adherence to the national iv-oral switch guideline. This resulted in a high switch-rate of $72 \%$.

The use of a computerized intervention has been investigated in a limited number of studies. Fisher et al. developed computerized interventions which automatically sent reminders to the physician, when an iv-oral switch was possible. Of the iv orders $21.6 \%$ were replaced by an oral agent, and $14 \%$ of the selected orders were discontinued [10]. However, this intervention was initiated on only 5 different categorized target drugs and adherence was low. Another study showed a decrease of iv use of levofloxacin and ciprofloxacin when displaying electronic alerts [9].

Beeler et al. [14] performed a prospective, controlled trial using an electronic reminder to stimulate iv-oral 
switch. Their study resulted in a decrease of total iv duration of $17.5 \%$ in the intervention group, and a switch-rate of $26.6 \%$. Unfortunately, they did not report on the amount of patients who were not eligible for switch, which could explain the difference with the adherence rate in our own study.

Our study has several strengths. First, we used a large patient population and physician response to the switch reminder was high (72\%). The advantage of our switch intervention lies in the possibilities of automatically identifying patients eligible for switch and alerting the physician. Besides the distribution of the switch-forms by the department secretary, no man-power is required for the continuous operation of the trigger tool, furthermore the educational program only takes 15 -min a week. This enables antimicrobial stewardship teams to focus on other stewardship activities. The implementation of more sophisticated electronic health record systems (EHRS), which provide electronic alerts, could contribute to an additional reduction of workload [14, 15].

A recent survey conducted in France showed that human resources needed to implement AST activities were estimated at 3.6 full-time equivalent (FTE) positions/ 1000 acute care beds for antibiotic/infectious disease lead supervisors, at $2.5 \mathrm{FTE} / 1000$ beds for pharmacists, and at $0.6 \mathrm{FTE} / 1000$ beds for microbiologists [16]. Most hospitals in the Netherlands have restricted manpower for AST and can only focus on a limited number of stewardship activities. Our intervention is free and effective and can contribute to a simple and successful implementation of an iv-oral switch program.

Second, our controlled, interrupted time series analysis design is more robust than (un)controlled before and after analyses or uncontrolled interrupted time series designs used in most stewardship studies [17]. We were able to identify a significant difference in intravenous usage of antibiotics $>72 \mathrm{~h}$ on the intervention wards (reduction 19\%). However, in the control wards a significant effect (reduction 6\%) was also found. This effect could possibly be contributed to the nationwide and local hospital attention for antimicrobial stewardship during the time of the study. Nevertheless, our iv-oral switch intervention was responsible for an additional effect of $13 \%$ reduction in iv antibiotic usage $>72 \mathrm{~h}$ in favor of the intervention wards.

Our study has some limitations. First, baseline characteristics of the intervention and control groups were not comparable. The intervention was implemented at internal medicine wards and the control wards represented all other hospital wards; the baseline percentage of intravenous antibiotic use for $>72 \mathrm{~h}$ was higher $(+0.7 \%)$ in the intervention group. Therefore, the effect of our intervention may not be transferable to all hospital wards. Second, our intervention was not fully effective, from all antibiotics eligible for switch, $28 \%$ were incorrectly not switched and due to organizational factors we were not able to implement the intervention during the weekend. This indicates that there is still ample room for improvement. Since physicians were asked to fill in reasons for not switching, these will be used to improve the educational program. Third, patients who received solely iv antibiotics could have been incorrectly excluded, while they may have been candidates for iv-oral switch therapy. Nowadays, with modern EHRS, more sophisticated algorithms that are based on general accepted switch criteria, and that incorporate both clinical, pharmaceutical and laboratory details could be developed.

Finally, this was a single center study, therefore, results may not be widely generalizable. However, the iv-oral switch algorithm was designed based upon commonly used clinical and laboratory criteria. With the help of an information technician the implementation in other hospitals with an electronic prescribing system should be possible.

\section{Conclusion}

This study showed the efficacy of an electronic trigger tool combined with targeted education on iv-oral switch. The intervention significantly decreased the amount of intravenously administered antibiotics used for $>72 \mathrm{~h}$ by $19 \%$.

This trigger tool enables hospital ASTs to implement a feasible and effective stand-alone iv-oral switch strategy, winning them valuable time to address more complicated issues of antimicrobial prescribing in daily practice.

\section{Additional files}

Additional file 1: Appendix 1. Electronic trigger tool algorithm to identify patients eligible for iv to oral switch. "high dose of a penicillin or cephalosporin only indicated for severe infection (e.g. endocarditis or meningitis) or an antibiotic class which is given only if no oral formulation is available (carbapenem). (JPEG $63 \mathrm{~kb}$ )

Additional file 2: Appendix 2. Protocol for iv to oral switch therapy. (DOCX $17 \mathrm{~kb}$ )

\section{Abbreviations \\ AST: Antimicrobial stewardship teams; EHRS: Electronic health record systems; FTE: Full-time equivalent; ITSA: Interrupted time series analysis; IV: Intravenous}

\section{Acknowledgements}

Not applicable.

Funding

Not applicable.

Availability of data and materials

The datasets used and/or analysed during the current study are available from the corresponding author on reasonable request. 


\section{Authors' contributions}

$\mathrm{MB}$ and JP analyzed and interpreted the patient data and wrote the manuscript. $\mathrm{AH}, \mathrm{AD}, \mathrm{MN}$ and $\mathrm{HF}$ were involved in developing the electronic trigger tool, implementing the intervention in the hospital, and data collection. TS and JS coordinated the study team. TS, JS and BJK were a major contributor in writing the manuscript. All authors edited, read and approved the manuscript.

\section{Ethics approval and consent to participate}

This study does not fall within the remit of the Medical Research involving Human Subjects Act (WMO). Therefore the study can be carried out in the Netherlands without an approval by an accredited research ethics committee and without explicit informed consent of the participants.

\section{Consent for publication}

Not applicable.

\section{Competing interests}

The authors declare that they have no competing interests.

\section{Publisher's Note}

Springer Nature remains neutral with regard to jurisdictional claims in published maps and institutional affiliations.

\section{Author details}

${ }^{1}$ Department of Internal Medicine and Infectious Diseases, Radboudumc, Nijmegen, the Netherlands. ${ }^{2}$ Department of Clinical Pharmacy, Canisius-Wilhelmina Hospital, Nijmegen, the Netherlands. ${ }^{3}$ Department of Internal Medicine, Canisius-Wilhelmina Hospital, Nijmegen, the Netherlands. ${ }^{4}$ Department of Medical Microbiology and Infectious Diseases, Canisius-Wilhelmina Hospital, Nijmegen, the Netherlands. ${ }^{5}$ Department of Intensive Care, Canisius-Wilhelmina Hospital, Nijmegen, the Netherlands.

Received: 20 March 2017 Accepted: 4 August 2017

Published online: 15 August 2017

\section{References}

1. Ahkee S, Smith S, Newman D, Ritter W, Burke J, Ramirez JA. Early switch from intravenous to oral antibiotics in hospitalized patients with infections: a 6-month prospective study. Pharmacotherapy. 1997;17:569-75.

2. Athanassa Z, Makris G, Dimopoulos G, Falagas ME. Early switch to oral treatment in patients with moderate to severe community-acquired pneumonia: a meta-analysis. Drugs. 2008;68:2469-81.

3. Sevinc F, Prins JM, Koopmans RP, Langendijk PN, Bossuyt PM, Dankert J, et al. Early switch from intravenous to oral antibiotics: guidelines and implementation in a large teaching hospital. J Antimicrob Chemother. 1999;43:601-6.

4. Handoko KB, van Asselt GJ, Overdiek JW. Preventing prolonged antibiotic therapy by active implementation of switch guidelines. Ned Tijdschr Geneeskd. 2004;148:222-6.

5. Oosterheert JJ, Bonten MJ, Schneider MM, Buskens E, Lammers JW, Hustinx WM, et al. Effectiveness of early switch from intravenous to oral antibiotics in severe community acquired pneumonia: multicentre randomised trial. BMJ. 2006:333:1193.

6. Schuts EC, Hulscher ME, Mouton JW, Verduin CM, Stuart JW, Overdiek HW, et al. Current evidence on hospital antimicrobial stewardship objectives: a systematic review and meta-analysis. Lancet Infect Dis. 2016;16(7):847-56.

7. Engel MF, Postma DF, Hulscher ME, Teding van Berkhout F, EmmelotVonk MH, Sankatsing S, et al. Barriers to an early switch from intravenous to oral antibiotic therapy in hospitalised patients with CAP. Eur Respir J. 2013;41:123-30

8. Schouten JA, Hulscher ME, Natsch S, Kullberg BJ, van der Meer JW, Grol RP. Barriers to optimal antibiotic use for community-acquired pneumonia at hospitals: a qualitative study. Qual Saf Health Care. 2007;16:143-9.

9. Hulgan T, Rosenbloom ST, Hargrove F, Talbert DA, Arbogast PG, Bansal P, et al. Oral quinolones in hospitalized patients: an evaluation of a computerized decision support intervention. J Intern Med. 2004;256:349-57.

10. Fischer MA, Solomon DH, Teich JM, Avorn J. Conversion from intravenous to oral medications: assessment of a computerized intervention for hospitalized patients. Arch Intern Med. 2003;163:2585-9.
11. Wagner AK, Soumerai SB, Zhang F, Ross-Degnan D. Segmented regression analysis of interrupted time series studies in medication use research. J Clin Pharm Ther. 2002;27:299-309.

12. Kontopantelis E, Doran T, Springate DA, Buchan I, Reeves D. Regression based quasi-experimental approach when randomisation is not an option: interrupted time series analysis. BMJ. 2015;350:h2750.

13. Buyle FM, Metz-Gercek S, Mechtler R, Kern W, Robays H, Vogelaers D, et al. Prospective multicentre feasibility study of a quality of care indicator for intravenous to oral switch therapy with highly bioavailable antibiotics. J Antimicrob Chemother. 2012;67:2043-6.

14. Beeler PE, Kuster SP, Eschmann E, Weber R, Blaser J. Earlier switching from intravenous to oral antibiotics owing to electronic reminders. Int J Antimicrob Agents. 2015:46:428-33.

15. Kim M, Song KH, Kim CJ, Song M, Choe PG, Park WB, et al. Electronic alerts with automated consultations promote appropriate antimicrobial prescriptions. Plos One. 2016;11:e0160551.

16. Le Coz P, Carlet J, Roblot F, Pulcini C. Human resources needed to perform antimicrobial stewardship teams' activities in French hospitals. Med Mal Infect. 2016;46:200-6.

17. McGregor JC, Furuno JP. Optimizing research methods used for the evaluation of antimicrobial stewardship programs. Clin Infect Dis. 2014; 59(Suppl 3):S185-92

\section{Submit your next manuscript to BioMed Central and we will help you at every step:}

- We accept pre-submission inquiries

- Our selector tool helps you to find the most relevant journal

- We provide round the clock customer support

- Convenient online submission

- Thorough peer review

- Inclusion in PubMed and all major indexing services

- Maximum visibility for your research

Submit your manuscript at www.biomedcentral.com/submit
) Biomed Central 